ARTICLE

Received 5 Feb 2013 | Accepted 12 Aug 2013 | Published 12 Sep $2013 \quad$ DOI: 10.1038/ncomms3431

\title{
Two-dimensional vanadyl phosphate ultrathin nanosheets for high energy density and flexible pseudocapacitors
}

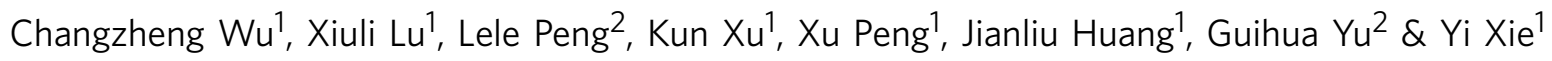

Two-dimensional materials have been an ideal material platform for constructing flexible ultrathin-film supercapacitors, offering great advantages of flexibility, ultra-thinness and even transparency. Exploring new two-dimensional pseudocapacitive materials with high electrochemical activity is needed to achieve flexible ultrathin-film supercapacitors with higher energy densities. Here we report an inorganic graphene analogue, $\alpha_{1}$-vanadyl phosphate ultrathin nanosheets with less than six atomic layers, as a promising material to construct a flexible ultrathin-film pseudocapacitor in all-solid-state. The material exhibits a high potential plateau of $\sim 1.0 \mathrm{~V}$ in aqueous solutions, approaching the electrochemical potential window of water $(1.23 \mathrm{~V})$. The as-established flexible supercapacitor achieves a high redox potential $(1.0 \mathrm{~V})$ and a high areal capacitance of $8,360.5 \mu \mathrm{F} \mathrm{cm}^{-2}$, leading to a high energy density of $1.7 \mathrm{mWh} \mathrm{cm}^{-2}$ and a power density of $5.2 \mathrm{~mW} \mathrm{~cm}^{-2}$.

\footnotetext{
${ }^{1}$ Hefei National Laboratory of Physical Sciences at the Microscale, University of Science and Technology of China, Hefei, Anhui 230026, China. ${ }^{2}$ Materials Science and Engineering Program and Department of Mechanical Engineering, The University of Texas at Austin, Austin, Texas 78712, USA. Correspondence and requests for materials should be addressed to Y.X. (email: yxie@ustc.edu.cn) or to G.Y. (email: ghyu@austin.utexas.edu).
} 
rapidly growing demand for portable consumer electronics, such as flexible displays, mobile phones and notebook computers, has greatly promoted the development of flexible energy devices in all-solid-state ${ }^{1-7}$. As the futuregeneration energy storage device, the flexible thin-film supercapacitor in all-solid-state offers the synergic benefits of flexibility, thinness and transparency ${ }^{2,8-12}$. Among the different types of supercapacitors, pseudocapacitors have the advantage of a highly electroactive surface of the electrode materials owing to fast redox reaction, and exhibit much higher energy density compared with electrical double-layer capacitors as well as higher power density compared with lithium-ion batteries ${ }^{13,14}$, holding great promise for realizing high-performance flexible ultrathinfilm supercapacitor in all-solid-state (FUSA) with pseudocapacitive behaviour ${ }^{15-17}$. Pursuing two-dimensional (2D) graphene-like materials with pseudocapacitive characteristics represents a promising direction to accomplish the flexible ultrathin-film pseudocapacitor in all-solid-state (FUPA) with higher energy density, and potentially excellent mechanical flexibility ${ }^{18-20}$. Moreover, obtaining the graphene-like pseudocapacitive materials not only requires the intrinsic layered structures that can be exfoliated into $2 \mathrm{D}$ ultrathin nanosheets, but also needs to possess a high electrochemical activity and high redox potential that can lead to higher energy density $\left(\mathrm{E} \sim \mathrm{V}^{2}\right)$. However, the development of graphene-like pseudocapacitive materials is still at its early stage, especially for the FUPAs with high power and energy densities. For example, the reversible redox Faradaic reactions occurred on the surface of layered $\mathrm{Ni}(\mathrm{OH})_{2}$ and $\mathrm{Co}_{3} \mathrm{O}_{4}$ exhibit the potential windows of only about 0.5 and $0.4 \mathrm{~V}$, respectively ${ }^{13,20}$, both of which are still much lower than the limited electrochemical window of the aqueous solution $(1.23 \mathrm{~V})^{21}$. Therefore, the discovery of new graphene-like pseudocapacitive materials with enhanced electrochemical performance is much needed for constructing FUSA with higher power and energy densities, vital for satisfying the practical applications.

Vanadyl phosphate $\left(\mathrm{VOPO}_{4}\right)$ has seen tremendous advances for layered materials with higher electrochemical performance ${ }^{22-24}$. Due to the enhanced inionicity of $(\mathrm{V}-\mathrm{O})$ bonds when $\left(\mathrm{PO}_{4}\right)^{3-}$ anion is introduced, $\mathrm{V}^{4+} / \mathrm{V}^{5+}$ redox couple of $\mathrm{VOPO}_{4}$ possesses the higher potential than that for simple vanadium oxide ${ }^{25}$. Layered $\mathrm{VOPO}_{4}$ has a high redox potential of $1.0 \mathrm{~V}$ versus normal hydrogen electrode (NHE), and thus it is promising to establish the pseudocapacitors with improved energy density (Supplementary Fig. S1 $)^{26,27}$. Furthermore, dehydrated vanadyl phosphate $\left(\mathrm{VOPO}_{4}\right.$ $2 \mathrm{H}_{2} \mathrm{O}$ ) has the characteristic layered structure, in which the sheets of $\mathrm{VOPO}_{4}$ form from vertex-sharing $\mathrm{VO}_{6}$ octahedra linking to phosphate $\mathrm{PO}_{4}$ tetrahedra. Between each $\mathrm{VOPO}_{4}$ layer, one water molecule coordinates with a vanadium atom through an oxygen atom and the other links adjacent layers together through weak hydrogen bonds (Supplementary Fig. S2) ${ }^{28}$. The presence of weak hydrogen bonds in $\mathrm{VOPO}_{4} \cdot 2 \mathrm{H}_{2} \mathrm{O}$ provides the feasible clue to the exfoliation of layered $\mathrm{VOPO}_{4} \cdot 2 \mathrm{H}_{2} \mathrm{O}$ into $\mathrm{VOPO}_{4}$ ultrathin nanosheets while maintaining the integrity of the in-plane structure. With the synergic advantages of high redox potential and the layered structure, the exfoliated $\mathrm{VOPO}_{4}$ ultrathin nanosheets could be a promising new $2 \mathrm{D}$ graphene-like material with greatly enhanced electrochemical properties. Although $\mathrm{VOPO}_{4}$ shows great potential for applications in flexible supercapacitors, its graphene-like material has long been unexplored in the past years, let alone the realization of the corresponding energy storage devices.

Here we report a simple 2-propanol-assisted ultrasonication method to effectively exfoliate bulk $\mathrm{VOPO}_{4} \cdot 2 \mathrm{H}_{2} \mathrm{O}$ into $\mathrm{VOPO}_{4}$ ultrathin nanosheets, with a thickness of less than six atomic layers, as a new graphene-like material. To fully explore the electrochemical performance of $\mathrm{VOPO}_{4}$, a $\mathrm{VOPO}_{4} /$ graphene hybrid film was layer-by-layer assembled to achieve both high planar conductivity and superior electrochemical performance. The FUPA based on the $\mathrm{VOPO}_{4}$ /graphene hybrid thin film demonstrated a high output voltage, a large specific capacitance and a high energy density, opening up opportunities for exploring new quasi-2D materials for flexible energy device with higher energy density.

\section{Results}

$\mathrm{VOPO}_{4}$ ultrathin nanosheet. The $\mathrm{VOPO}_{4}$ ultrathin nanosheets were achieved by exfoliation of bulk $\mathrm{VOPO}_{4} \cdot 2 \mathrm{H}_{2} \mathrm{O}$ through a simple ultrasonication method in 2-propanol with a short reaction time of $15 \mathrm{~min}$. For bulk $\mathrm{VOPO}_{4} \cdot 2 \mathrm{H}_{2} \mathrm{O}, \mathrm{VOPO}_{4}$ layers are linked together by hydrogen bonds from the interaction between $\mathrm{H}_{2} \mathrm{O}$ molecular and $\mathrm{VOPO}_{4}$ layers. As one kind of weak intermolecular force, hydrogen bond is very sensitive to the applied external force ${ }^{29}$. For example, the applied strong ultrasonication provides the powerful force to trigger the breaking of hydrogen bonds. In our case, the exfoliation process from bulk $\mathrm{VOPO}_{4}$ $2 \mathrm{H}_{2} \mathrm{O}$ to $\mathrm{VOPO}_{4}$ graphene-like material was conducted in 2propanol solution, in that 2-propanol as a secondary alcohol is more suitable than primary alcohols as the dispersant due to the lower reactivity of hydroxyl group with the $\mathrm{VOPO}_{4}$ layer matrix (Supplementary Fig. S3). The interaction force of hydrogen bonds between 2-propanol and $\mathrm{H}_{2} \mathrm{O}$ further promoted the $\mathrm{H}_{2} \mathrm{O}$ molecules extracted from the interlayer space of bulk $\mathrm{VOPO}_{4} \cdot 2 \mathrm{H}_{2} \mathrm{O}$. (Fig. 1a). The obtained $\mathrm{VOPO}_{4}$ ultrathin nanosheets were homogeneously dispersed in solution with high stability for over several months.

The structural transformation process during the exfoliation could be revealed by the characterizations of X-ray powder diffraction (XRD) patterns and Raman spectra, which performed on the transferrable $\mathrm{VOPO}_{4}$ thin films. The XRD characterization of vacuum-filtration assembled films of samples at different sonication time is shown in Fig. 1b. The XRD pattern at the initial sonication process can be readily indexed into the tetragonal $\mathrm{VOPO}_{4} \cdot 2 \mathrm{H}_{2} \mathrm{O}$ with the standard JCPDS card No.84-0111 ( $\mathrm{VOPO}_{4} \cdot 2 \mathrm{H}_{2} \mathrm{O}$, space group $\mathrm{P} 4 /$ $\mathrm{nmm}, a=6.202 \AA, b=6.202 \AA, c=7.410 \AA)$. As the strong ultrasonication proceeds, the (001) peak became relatively stronger compared with other XRD peaks, revealing that the applied sonication waves triggered the structural arrangements and enhanced the $c$ axis orientation for the formation process of high-quality 2D nanosheets. Furthermore, the (001) peak of $\mathrm{VOPO}_{4} \cdot 2 \mathrm{H}_{2} \mathrm{O}$ gradually shifted from lower $2 \theta\left(11.74^{\circ}\right)$ to a higher one $\left(13.5^{\circ}\right)$ during the sonication process, indicating the decrease of the interlayer spacing distance from $\mathrm{VOPO}_{4} \cdot 2 \mathrm{H}_{2} \mathrm{O}$ to the final $\mathrm{VOPO}_{4}$ ultrathin nanosheets as a result of the escape of molecular $\mathrm{H}_{2} \mathrm{O}$ from $\mathrm{VOPO}_{4}$ interlayers.

In addition, Raman spectra shown in Fig. 1c further verified structural evolutions during the exfoliation process. The bands at $937 \mathrm{~cm}^{-1}$ were assigned to the symmetric O-P-O stretching modes $^{30,31}$. With the elongated exfoliation time, the red shift of Raman peaks of O-P-O stretching mode was more noticeable. Obviously, there was a strong microstructural correlation between symmetry of $\mathrm{O}-\mathrm{P}-\mathrm{O}$ stretching modes with the hydrogen bonding among the interlayered $\mathrm{H}_{2} \mathrm{O}$ molecular. With the breaking of hydrogen bonds from the oxygen atoms of the $\mathrm{P}-\mathrm{O}$ bond in $\mathrm{VOPO}_{4}$, the mitigation of the steric hindrance would facilitate the occurrence of the $\mathrm{O}-\mathrm{P}-\mathrm{O}$ stretching modes with lower energy. However, the peaks related to the symmetric bending vibrations of $\mathrm{O}-\mathrm{P}-\mathrm{O}, \mathrm{V}-\mathrm{O}$ and $\mathrm{V}=\mathrm{O}$ stretching mode demonstrated little shift with no obvious peak position evolution (Supplementary Fig. S4). The Raman results confirmed that the 
a

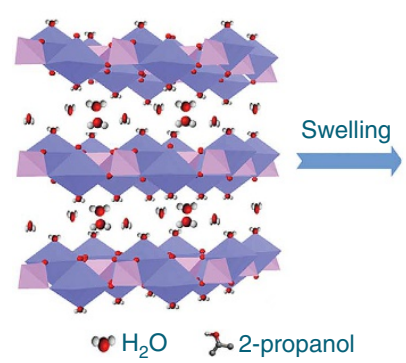

b

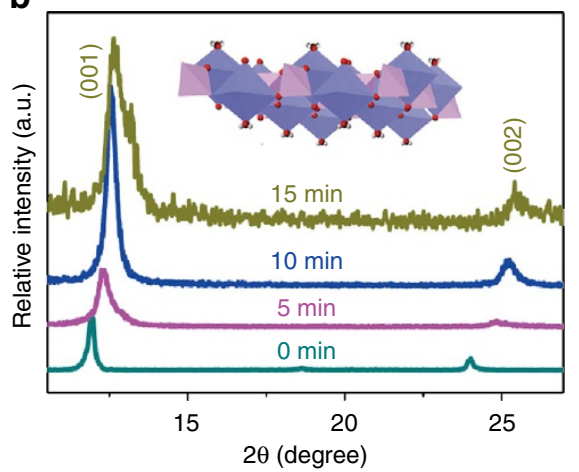

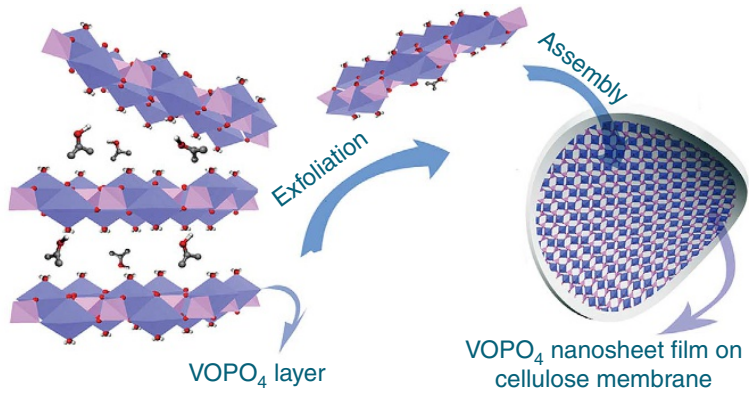

C

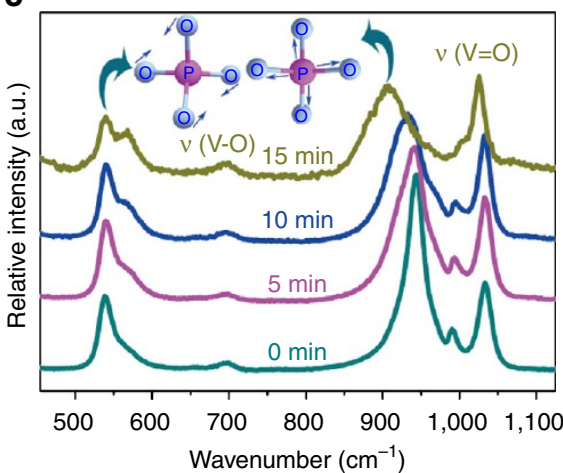

Figure 1 | Formation of $\mathbf{V O P O}_{\mathbf{4}}$ ultrathin nanosheets. (a) Schematic illustration for 2-propanol-assisted exfoliation process from bulk $\mathrm{VOPO} \cdot \mathrm{H}_{4} \cdot 2 \mathrm{H}_{2} \mathrm{O}$ to graphene-like $\mathrm{VOPO}_{4}$ nanosheets. During the process of ultrasonication in 2-propanol solution, the $\mathrm{VOPO}_{4}$ layers were swelled and interlayered $\mathrm{H}_{2} \mathrm{O}$ molecules effused, resulting in the $\mathrm{VOPO}_{4}$ ultrathin nanosheets. $(\mathbf{b}, \mathbf{c})$ XRD and Raman spectra of samples for the elongated ultrasonication time of $0,5,10$ and $15 \mathrm{~min}$, respectively. The insets in $\mathbf{c}$ are the symmetric bending (left) and stretching (right) modes of O-P-O, respectively.
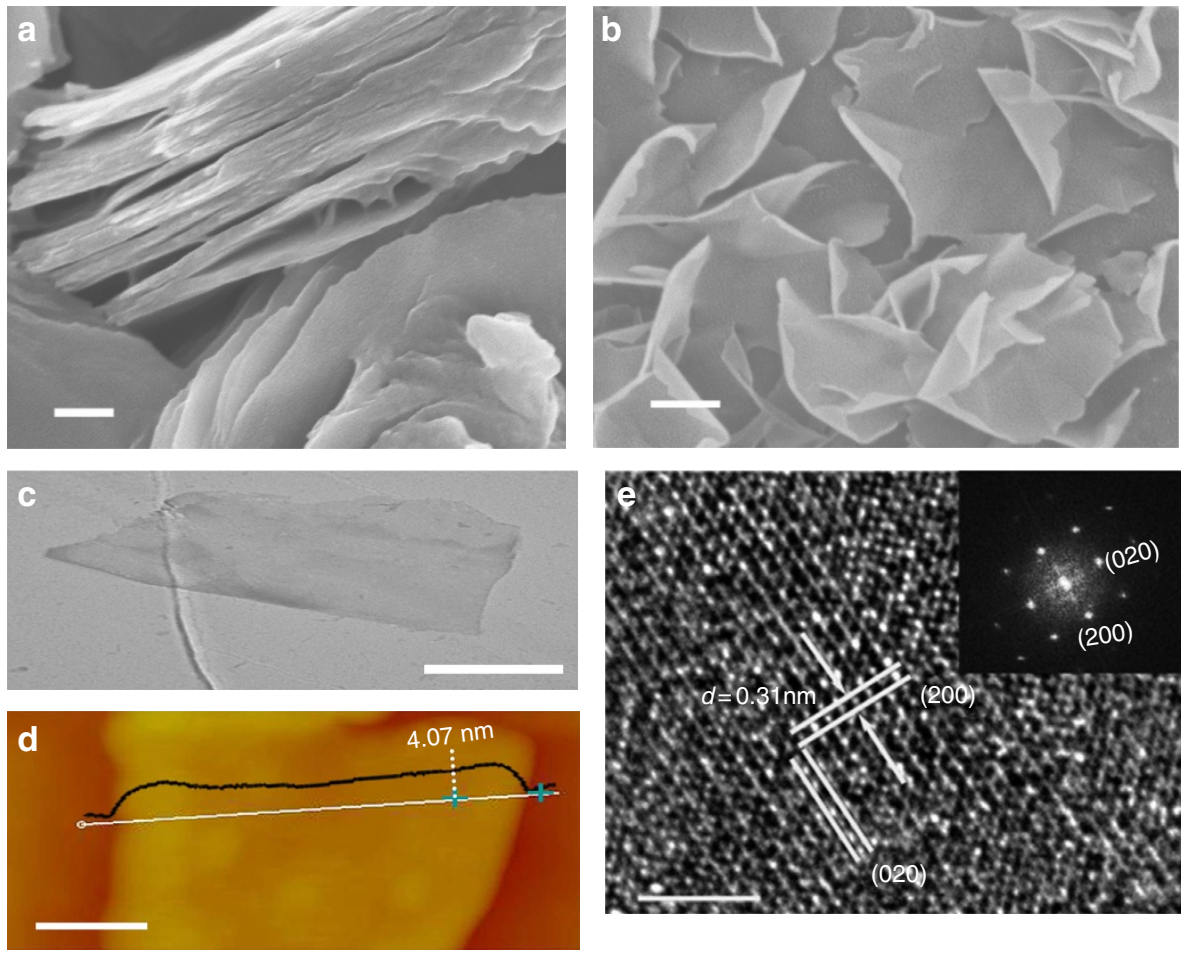

Figure 2 | Microstructural characterization of ultrathin nanosheets. (a) Field emission scanning electron microscopy image of bulk $\mathrm{VOPO} \cdot 2 \mathrm{H}_{2} \mathrm{O}$ precursors, where the stacked layers can be clearly seen. Scale bar, $200 \mathrm{~nm}$. (b) The SEM image of exfoliated VOPO 4 ultrathin nanosheets with warped edges exhibiting the ultrathin features. Scale bar, $200 \mathrm{~nm}$. (c) TEM image of a typical ultrathin nanosheet. Scale bar, $500 \mathrm{~nm}$. (d) Atomic force microscopy image of a typical nanosheet with a thickness of $4.07 \mathrm{~nm}$, corresponding to less than six atomic layers. Scale bar, $100 \mathrm{~nm}$. (e) High-resolution TEM (HR-TEM) image of a typical nanosheets showing the lattice fringes of (200) and (020) planes, and the inset is the corresponding fast Fourier transform patterns of the same area in the HR-TEM image. Scale bar, $2 \mathrm{~nm}$. 
exfoliated $\mathrm{VOPO}_{4}$ nanosheets maintained the integrity of the in-plane $\mathrm{VOPO}_{4}$ structure without obvious structural deformations.

To unravel the microscopic outlook and structural crystallinity of exfoliated 2D $\mathrm{VOPO}_{4}$ nanosheets, microstructural characterizations were performed. Scanning electron microscopy (SEM) images of the precursor $\mathrm{VOPO}_{4} \cdot 2 \mathrm{H}_{2} \mathrm{O}$ and graphene-like $\mathrm{VOPO}_{4}$ nanosheets were taken and compared as shown in Fig. 2 and Supplementary Fig. S5. The bulk $\mathrm{VOPO}_{4} \cdot 2 \mathrm{H}_{2} \mathrm{O}$ exhibited the typical layered structure, of which the layers were tightly stacked. In contrast, SEM image of the exfoliated products after 15 min ultransonication (Fig. 2b) shows the morphology of ultrathin nanosheets with a typical size ranging from $400 \mathrm{~nm}$ to several micrometres. Transmission electron microscopy (TEM) image of the exfoliated $\mathrm{VOPO}_{4}$ nanosheet in Fig. $2 \mathrm{c}$ reveals a freestanding, sheet-like morphology with a lateral size of $\sim 1 \mu \mathrm{m}$, and the nearly transparent feature implies the ultrathin thickness of exfoliated nanosheets. Atomic force microscopy image in Fig. 2d was taken to further evaluate the thickness of the $\mathrm{VOPO}_{4}$ nanosheet. The measured height was $\sim 4.07 \mathrm{~nm}$, denoting that the nanosheet was comprised of 5-6 single layers, given that the $c$ parameter of the $\mathrm{VOPO}_{4} \cdot 2 \mathrm{H}_{2} \mathrm{O}$ is $7.410 \AA$. The corresponding HR-TEM image and fast Fourier transform pattern are shown in Fig. 2e, demonstrating that the exfoliated sheets were single crystalline with [001] preferential orientation. The interplanar distance of $0.31 \mathrm{~nm}$ fits well with the plane distance of $d_{200}$ and $d_{202}$, respectively. The orientation angle values $90^{\circ}$ of these two planes of (200) and (020) in HR-TEM image and fast Fourier transform pattern was consistent with those calculated from tetragonal crystallographic parameters of $\mathrm{VOPO}_{4} \cdot 2 \mathrm{H}_{2} \mathrm{O}$. These characterization results showed that $\mathrm{VOPO}_{4} \cdot 2 \mathrm{H}_{2} \mathrm{O}$ was successfully exfoliated into ultrathin $\mathrm{VOPO}_{4}$ nanosheets that exhibited good crystallinity and high $c$ axis orientation, providing strong basis for further assembly of $\mathrm{VOPO}_{4}$ nanosheets into large-area practical energy storage devices.

2D hybrid thin film. The bulk $\mathrm{VOPO}_{4}$ is well known as a highperformance electrochemical material with a high redox voltage under aqueous solution of $1.0 \mathrm{~V}$ versus NHE, approaching the electrochemical window of water $(1.23 \mathrm{~V})^{21}$, which entails the great fascination for the construction of high-efficiency energy storage devices. (Supplementary Fig. S1) In our work, $\mathrm{VOPO}_{4}$ ultrathin nanosheets with $<6$ atomic layers, as a new inorganic graphene-like material, provide an ideal 2D material platform to investigate their electrochemical behaviour, and to give the feasibility to construct the FUPA. Because of the poor conductivity of $\mathrm{VOPO}_{4}$ nanosheet, a layer-by-layer strategy was adopted to assemble a $\mathrm{VOPO}_{4} /$ graphene hybrid film, and the hybrid thin film exhibits advantageous features of being uniform, compact and nearly transparent (Fig. 3a). The hybrid film could be readily transferred and free-standing due to its high-quality film structure. Fig. $3 \mathrm{~b}$ is the cross-section SEM image of the hybrid film, showing the compact layer-by-layer structure with the thickness of $\sim 90 \mathrm{~nm}$ thick. The inset in Fig. $3 \mathrm{c}$ is the SEM image of the smooth hybrid film, where the graphene was underneath $\mathrm{VOPO}_{4}$ layers as illustrated by the white arrows. The combined analyses of the TEM and XRD results further reveal that the as-formed $\mathrm{VOPO}_{4} /$ graphene hybrid film was the stacking of serial layers of chemically integrated $\mathrm{VOPO}_{4}$ nanosheets on graphene sheets a

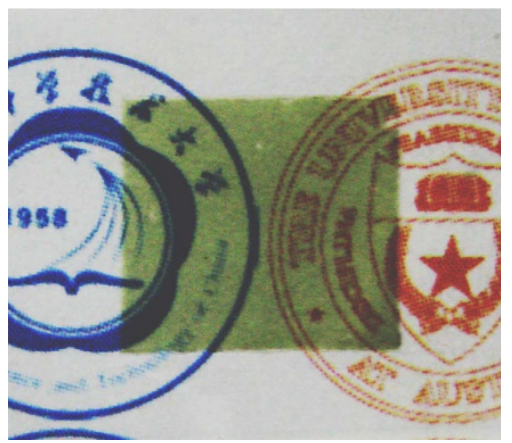

C

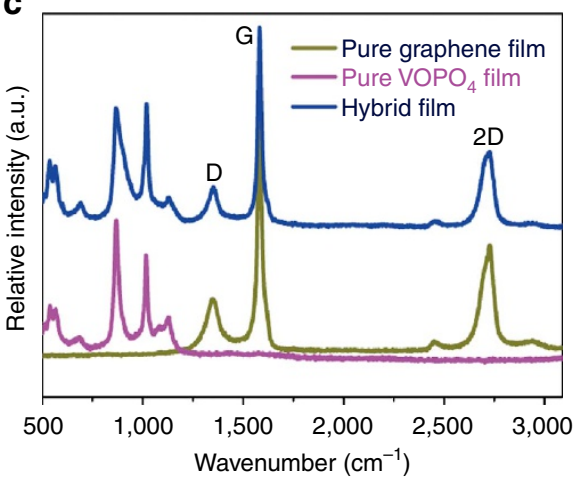

b

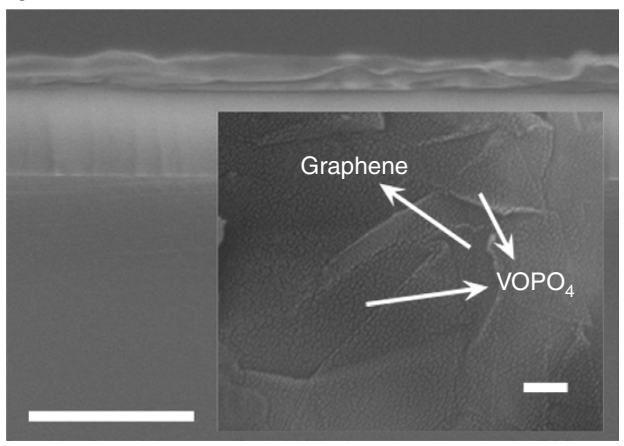

d

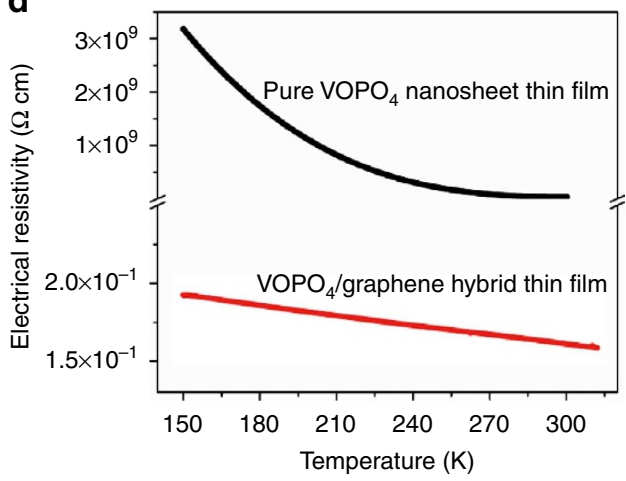

Figure 3 | Hybrid thin film of 2D VOPO $\mathbf{4}_{4}$ and graphene. (a) Demonstration of $\mathrm{VOPO}_{4} /$ graphene hybrid thin film on the surface of PET substrate, exhibiting transparent feature. (b) The cross-section SEM image of the hybrid thin film with the thickness of $\sim 90 \mathrm{~nm}$. The surface of the hybrid film, of which the graphene lies under the $\mathrm{VOPO}_{4}$ layers as indicated by the white arrows, is shown in the inset. Scale bar, $500 \mathrm{~nm}$; Inset scale bar, $100 \mathrm{~nm}$. (c) The Raman spectra of graphene film (brown line), $\mathrm{VOPO}_{4}$ film (red line) and $\mathrm{VOPO}_{4} /$ graphene hybrid film (blue line), respectively. (d) The temperature dependence of planar resistivity of the $\mathrm{VOPO}_{4}$ /graphene hybrid film and pure $\mathrm{VOPO}_{4}$ nanosheet thin film, where the incorporation of graphene greatly enhanced the electrical conductivity in the $\mathrm{VOPO}_{4} /$ graphene hybrid film. 
exhibiting the highly $c$ axis crystalline orientation, in which the $\mathrm{VOPO}_{4}$ nanosheets integrated on the graphene sheet as the building units and they assembled in stacks to form the final $\mathrm{VOPO}_{4}$ /graphene hybrid films (Supplementary Figs S6 and S7). To better understand the layer-by-layer assembly of $\mathrm{VOPO}_{4}$ nanosheets with graphene, Fig. $3 \mathrm{c}$ shows the Raman spectra of $\mathrm{VOPO}_{4}$ film, graphene film and $\mathrm{VOPO}_{4} /$ graphene hybrid film, respectively. The brown line is the Raman spectrum of graphene, which possesses two pronounced peaks at $1,580 \mathrm{~cm}^{-1}(\mathrm{G})$ and $2,700 \mathrm{~cm}^{-1}(2 \mathrm{D})$ of graphene. The stronger intensity of $\mathrm{G}$ peak shows that the synthesized graphene is of good quality and high crystallinity and the weaker D peak at $\sim 1,347 \mathrm{~cm}^{-1}$ corresponds to the lower disordering ${ }^{32-34}$. The red line represents the Raman spectrum of $\mathrm{VOPO}_{4}$ nanosheets film with characteristic peaks of $\mathrm{VOPO}_{4}$ at $\sim 866 \mathrm{~cm}^{-1}$ and $1,019 \mathrm{~cm}^{-1}$ corresponding to the symmetric stretching vibration $v_{1}\left(\mathrm{PO}_{4}\right)$ and $\mathrm{V}=\mathrm{O}$ stretching mode. The Raman spectrum of $\mathrm{VOPO}_{4} /$ graphene hybrid film (blue line) in Fig. $3 \mathrm{c}$ had the characteristic peaks of both $\mathrm{VOPO}_{4}$ nanosheets and graphene with homogeneous distribution. The $\mathrm{VOPO}_{4}$ /graphene hybrid films were of high-quality with uniform, compact, free-standing and high transparency features.

In addition, hybrid $\mathrm{VOPO}_{4}$ /graphene films exhibited greatly enhanced electrical properties. The hybrid film can be transferred onto various target substrates (Supplementary Fig. S8) for electrical measurements. Temperature dependence of planar electrical resistivity of the $\mathrm{VOPO}_{4} /$ graphene hybrid film was carried out as shown in Fig. 3d. As can be seen, the hybrid film possessed good conductivity in a wide temperature range from 150 to $300 \mathrm{~K}$ with slight resistivity change from $1.9 \times 10^{-1} \Omega \mathrm{cm}$ $(150 \mathrm{~K})$ to $1.6 \times 10^{-1} \Omega \mathrm{cm}(300 \mathrm{~K})$, which is eight orders of magnitude lower than that of pure $\mathrm{VOPO}_{4}$ nanosheet thin film $\left(3.0 \times 10^{7} \Omega \mathrm{cm}\right.$ at $\left.300 \mathrm{~K}\right)$. With the $\mathrm{VOPO}_{4}$ nanosheets integrated on the graphene layers, the $2 \mathrm{D} \mathrm{VOPO}_{4}$ /graphene hybrid film possessed the much improved electrical conductivity, making possible embodiment of outstanding electrochemical performance of $\mathrm{VOPO}_{4}$ nanosheets.

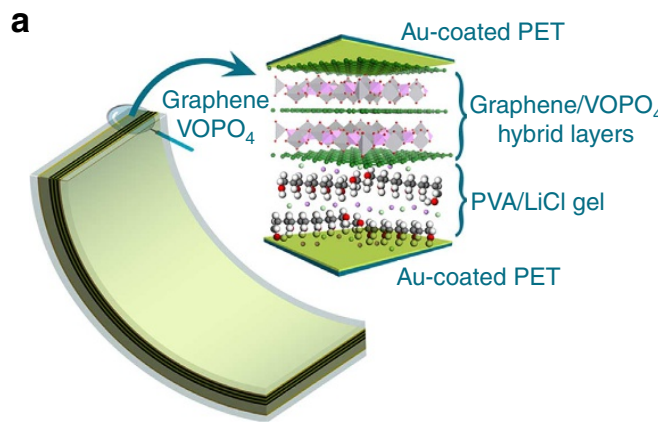

C

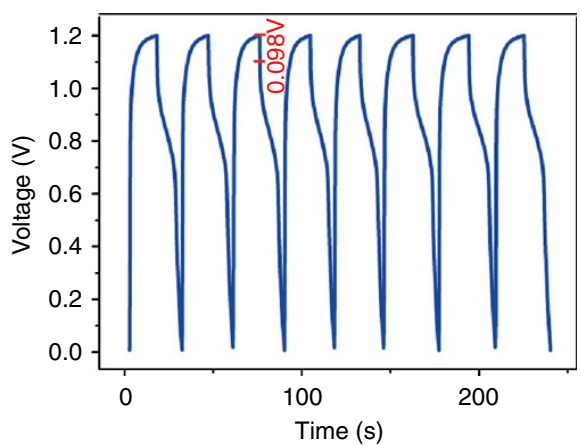

e

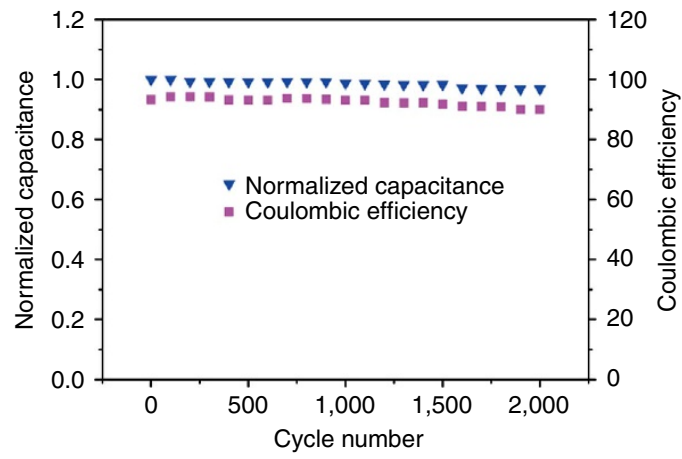

b

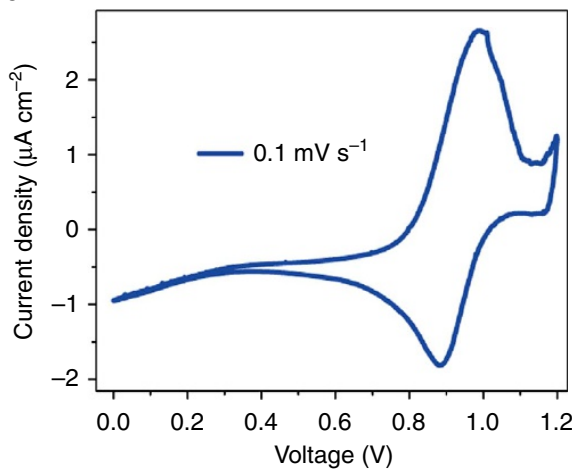

d

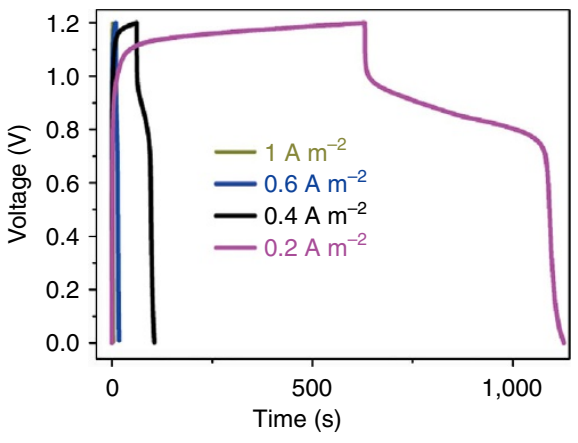

f

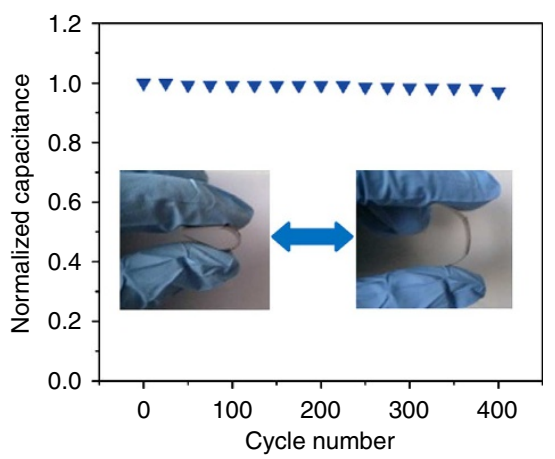

Figure 4 | Electrochemical performance. (a) Schematic illustration of an as-fabricated flexible ultrathin-film pseudocapacitor, in which the VOPO 4 graphene hybrid layers function as the working electrode and PVA/LiCl gel functions as the electrolyte. (b) $\mathrm{CV}$ curves of VOPO$/$ /graphene hybrid film in PVA/LiCl electrolyte from 0 to $1.2 \mathrm{~V}$ at $0.1 \mathrm{mVs}^{-1}$. (c) Galvanostatic cycling behaviour of the as-fabricated FUPA and its IR drop. (d) Galvanostatic chargedischarge curves at different current density of $0.2,0.4,0.6$ and $1 \mathrm{Am}^{-2}$. (e) Cycle test of the VOPO 4 /graphene hybrid film-based FUPA over 2,000 cycles. (f) Cycling stability of the FUPA under repeated bending/extending deformation. 
FUPA performance. To further study the electrochemical properties of the $\mathrm{VOPO}_{4}$ graphene-like materials, a FUPA based on $2 \mathrm{D} \mathrm{VOPO}_{4} /$ graphene hybrid film was fabricated. The FUPA devices were configured and fabricated according to the typical principles of flexiblity, ultra-thinness and all-solid-state, as illustrated in Fig. 4a. In this regard, $\mathrm{VOPO}_{4} /$ graphene hybrid film was transferred onto the gold-coated polyethylene terephthalate (PET) sheet as the working electrode. The polyvinyl alcohol (PVA)/LiCl was introduced as the solid electrolyte and separator to prevent potential dissolving of active material of $\mathrm{VOPO}_{4}$ nanosheets. Note that the salt $\mathrm{LiCl}$ could not be easily crystallized out during the process of gel drying and could provide a neutral $\mathrm{pH}$ medium, which makes $\mathrm{PVA} / \mathrm{LiCl}$ an excellent electrolyte for vanadium-involved solid-state pseudocapacitors ${ }^{35}$. The asassembled FUPA exhibited excellent electrochemical performance. Figure 4 summarizes the electrochemical performances of the as-fabricated FUPA based on $\mathrm{VOPO}_{4} /$ graphene utrathin film with a thickness of $\sim 90 \mathrm{~nm}$. Figure $4 \mathrm{~b}$ shows the cyclic voltammetry (CV) curve in the PVA/LiCl electrolyte from 0 to $1.2 \mathrm{~V}$ at scan rate of $0.1 \mathrm{mV} \mathrm{s}^{-1}$. The obvious redox current peaks at $\sim 1.1 \mathrm{~V}$ (oxidation peak) and $0.9 \mathrm{~V}$ (reduction peak) in the $\mathrm{CV}$ plots correspond to the reversible pseudocapacitive reactions of $\mathrm{VOPO}_{4}$ nanosheets with $\mathrm{Li}^{+}$, which can be represented by the following equation:

$$
\mathrm{VOPO}_{4}+x \mathrm{Li}^{+}+x e^{-} \rightarrow \mathrm{Li}_{x} \mathrm{VOPO}_{4}
$$

The slight internal resistance (IR) drop of $0.098 \mathrm{~V}$ observed in Fig. 4c implied a small intrinsic series resistance of $\mathrm{VOPO}_{4} /$ graphene hybrid film. Figure $4 \mathrm{c}$ shows the galvanostatic chargedischarge curves of the $\mathrm{VOPO}_{4} /$ graphene-based FUPA, from which the specific capacitance is calculated to be $8,360.5 \mu \mathrm{F} \mathrm{cm}^{-2}$ at current density of $0.2 \mathrm{Am}^{-2}$ (Supplementary Tables S1 and S2). Our areal capacitance of $8,360.5 \mu \mathrm{F} \mathrm{cm}^{-2}$ (that is, $928.9 \mathrm{~F} \mathrm{~cm}^{-3}$ ) is the highest value among all reported FUSAs ${ }^{18-20,36}$. The electrochemical performance of $2 \mathrm{D} \mathrm{VOPO}_{4} /$ graphene hybrid thin film is superior to that of bulk $\mathrm{VOPO}_{4}$, that of pure $2 \mathrm{D} \mathrm{VOPO}_{4}$ nanosheets and even to that of other crystal forms of $\mathrm{VOPO}_{4}$. (Supplementary Figs S9-S12). Moreover, the FUPAs fabricated with higher mass loading of active materials (thickness up to $\sim 10 \mu \mathrm{m}$ ) can still maintain good electrochemical performance, in which the areal capacitance increased 72 times when the thickness of electrode increased from $\sim 90 \mathrm{~nm}$ to $\sim 10 \mu \mathrm{m}$. (Supplementary Fig. S13). Employing pseudocapacitor in-plane, micro-electrodes can potentially further enhance the performance of 2D hybrid VOPO4/graphene thin film, as it could mitigate the issues from contact resistance and electrolyte ions diffusion in the case of much higher mass loading ${ }^{10,37}$. Furthermore, the discharge voltage up to $\sim 1.0 \mathrm{~V}$ is a significant step for constructing high energy density supercapacitors. Our reported discharge voltage up to $1.0 \mathrm{~V}$ is a fairly high value compared with previously reported supercapacitors in the aqueous electrolytes ${ }^{13-20}$. The high specific capacitance $\left(8,360.5 \mu \mathrm{Fcm}^{-2}\right)$ and redox voltage (up to $1.0 \mathrm{~V}$ ) lead to the ultrahigh energy density of $1.7 \mathrm{mWh} \mathrm{cm}^{-2}$ and power density of $5.2 \mathrm{~mW} \mathrm{~cm}^{-2}$. These are the highest values among the reported FUSAs as shown in Fig. 5 (Supplementary Tables S1 and S2). The improved energy density of FUSA with its high power density would make it more competitive for practical applications of energy storage devices ${ }^{38}$.

As shown in Fig. 4e, the as-fabricated FUPA had an excellent cycle life, as after 2,000 charge-discharge cycles specific capacitance retained $96 \%$ of the initial capacitance and Columbic efficiency was kept at $\sim 90 \%$, demonstrating excellent long-term cycling stability. Figure $4 \mathrm{f}$ demonstrates the excellent flexibility of the as-fabricated FUPA with negligible degradation of the specific capacitance even after 400 bending cycles. In short, the graphenelike $\mathrm{VOPO}_{4}$ materials have shown the fascinating electrochemical

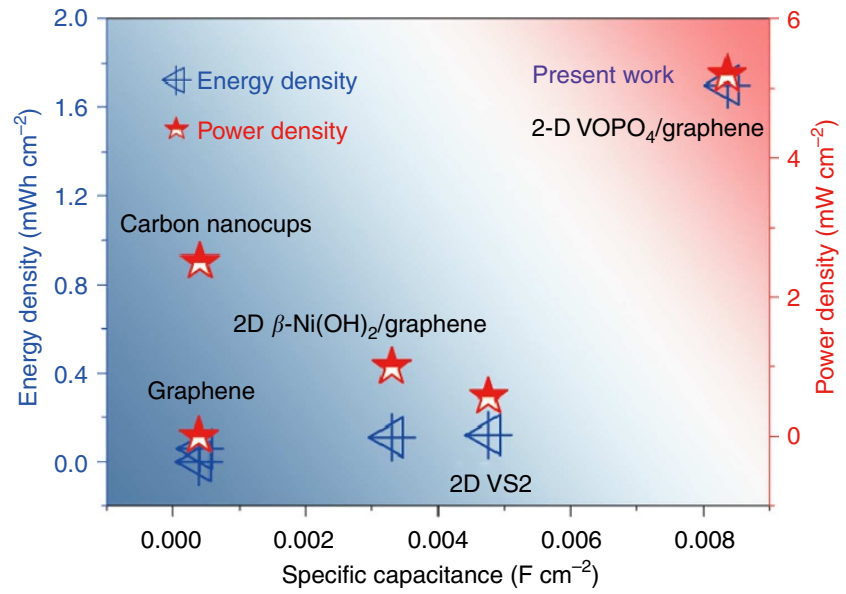

Figure 5 | Performance comparison of various materials. Our FUPA based on $2 \mathrm{D} \mathrm{VOPO}_{4} /$ graphene hybrid thin film showing the best performance of energy density compared with the reported flexible ultrathin-film supercapacitors in all-solid-state for graphene ${ }^{18}$, carbon nanocups ${ }^{36}$ $\mathrm{Ni}(\mathrm{OH})_{2}$ (ref. 20) and 2D VS (ref. 19).

and mechnical characteristics for practical applications in highperformance, flexible energy storage devices catering for portable and flexible electronics.

\section{Discussion}

In summary, $\mathrm{VOPO}_{4}$ ultrathin nanosheets with less than six atomic layers, as a new inorganic graphene-like material, have been reported for the first time. The as-fabricated FUPAs based on hybrid structure of the newly found $\mathrm{VOPO}_{4}$ ultrathin nanosheets and graphene sheets exhibit extremely high specific capacitance (up to $8,360.5 \mu \mathrm{F} \mathrm{cm}^{-2}$ ), high redox voltage (up to $1 \mathrm{~V}$ ), long cycle life (more than 2,000 cycles) and excellent flexibility, leading to an ultrahigh energy density of $1.7 \mathrm{mWh} \mathrm{cm}^{-2}$ and a power density of $5.2 \mathrm{~mW} \mathrm{~cm}^{-2}$. Our findings represent a promising direction and a significant step towards exploring new quasi-2D materials for flexible energy device with higher energy density in the near future.

\section{Methods}

Synthesis of bulk VOPO $\mathbf{P}_{\mathbf{4}} \cdot \mathbf{2 H}_{\mathbf{2}} \mathbf{O}$. Bulk $\mathrm{VOPO}_{4} 2 \mathrm{H}_{2} \mathrm{O}$ was obtained according to a simple method reported in the previous literature ${ }^{39}$. The mixture, including $\mathrm{V}_{2} \mathrm{O}_{5}$ $(4.8 \mathrm{~g}), \mathrm{H}_{3} \mathrm{PO}_{4}(85 \% 26.6 \mathrm{ml})$ and $\mathrm{H}_{2} \mathrm{O}(115.4 \mathrm{ml})$, was refluxed at $110^{\circ} \mathrm{C}$ for $16 \mathrm{~h}$. Thereafter, the system was permitted cool down to room temperature. The yellow precipitate was finally collected by filtration and washed several times with water and acetone. The resulting sample was dried in vacuum at $60^{\circ} \mathrm{C}$ for $3 \mathrm{~h}$.

Assembly of the hybrid VOPO4/graphene film. The graphene used in our experiments was synthesized according to the procedure used in previous studies ${ }^{40,41}$ The $\mathrm{VOPO}_{4} /$ graphene hybrid film was assembled by a layer-by-layer strategy. Specifically, the $\mathrm{VOPO}_{4}$ and graphene dispersion were vacuum filtered in turns over a cellulose membrane with $0.22 \mu \mathrm{m}$ pore size to form a homogeneous thin film. The thickness of the hybrid thin film can be tuned by changing the filtrated amount of the $\mathrm{VOPO}_{4}$ and graphene dispersion.

Preparation of gel electrolyte. Four grams of PVA powder was put into $40 \mathrm{ml}$ distilled water with stirring at $95^{\circ} \mathrm{C}$ (ref. 35). When the PVA powder was completely dissolved, $8.5 \mathrm{~g} \mathrm{LiCl}$ was added into the solution under vigorous stirring. After the LiCl/PVA turned to into a transparent and clear gel, it was cooled down at room temperature. At last, the $\mathrm{LiCl}$ gel electrolyte was prepared.

Exfoliation of bulk precursor into graphene-like material. Bulk $\mathrm{VOPO}_{4} \cdot 2 \mathrm{H}_{2} \mathrm{O}$ $(30 \mathrm{mg}$ ) was dispersed in a sealed glass bottle with $25 \mathrm{ml}$ 2-propanol and then the dispersion was ultrasonicated in iced water for $15 \mathrm{~min}$ forming the $\mathrm{VOPO}_{4}$ ultrathin nanosheets. The resultant yellow suspension was collected by vacuum filtration with a $0.22-\mu \mathrm{m}$ pore size cellulose membrane, forming a homogeneous thin film, which can be easily transferred to arbitrary substrates by pressing another side of the cellulose membrane. 
Fabrication of flexible supercapacitors in all-solid-state. FUPA devices typically include the working electrode, counter electrode, current collector and the flexible substrate. $\mathrm{VOPO}_{4} /$ graphene hybrid film was transferred onto the goldcoated PET sheet as the working electrode, in which the mass percentage of $\mathrm{VOPO}_{4}$ in the electrodes was $\sim 65 \%$ measured by inductively coupled plasma emission spectroscopy. The gold-coated PET sheet was used as the counter electrode; gold-coated PET sheet served dual functions as both the current collector and the flexible substrate. Meanwhile, PVA/LiCl was introduced as the solid electrolyte and separator to prevent the possible dissolving of the active material of $\mathrm{VOPO}_{4}$ nanosheets. The whole device was sealed carefully by the common transparent tape to make sure the device system is at a stable state.

Material characterizations. XRD was performed on a Philips X, Pert Pro Super diffractometer with $\mathrm{Cu} K \alpha$ radiation $(\lambda=1.54178 \AA)$. Raman spectra were recorded at room temperature with a LABRAM-HR confocal laser micro Raman spectrometer $750 \mathrm{~K}$ with a laser power of $0.5 \mathrm{~mW}$. The TEM images were taken on a JEOL2010 transmission electron microscope at an acceleration voltage of $200 \mathrm{kV}$. The field emission scanning electron microscopy images were obtained on a JEOL JSM6700F SEM. High-resolution TEM images were taken on a JEOL-2010 microscope at an accelerating voltage of $200 \mathrm{kV}$. Tapping-mode atomic force microscopy images were taken on a DI Innova Multimode SPM platform. Electrochemical performance of the supercapacitor was studied in a two-electrode system by $\mathrm{CV}$ and galvanostatic charge-discharge at an electrochemical station (CHI660B). Potential range set for galvanostatic charge-discharge tests was from 0 to $1.2 \mathrm{~V}$.

\section{References}

1. El-Kady, M. F., Strong, V., Dubin, S. \& Kaner, R. B. Laser scribing of highperformance and flexible graphene-based electrochemical capacitors. Science 335, 1326-1330 (2012).

2. Pushparaj, V. L. et al. Flexible energy storage devices based on nanocomposite paper. Proc. Natl Acad. Sci. USA 104, 13574-13577 (2007).

3. Nishide, H. \& Oyaizu, K. Toward flexible batteries. Science 319, 737-738 (2008).

4. Li, N., Chen, Z., Ren, W., Li, F. \& Cheng, H.-M. Flexible graphene-based lithium ion batteries with ultrafast charge and discharge rates. Proc. Natl Acad. Sci. 109, 17360-17365 (2012)

5. Fu, Y. et al. Fiber Supercapacitors utilizing pen ink for flexible/wearable energy storage. Adv. Mater. 24, 5713-5718 (2012).

6. Jia, X. et al. High-performance flexible lithium-ion electrodes based on robust network architecture. Energy Environ. Sci. 5, 6845-6849 (2012).

7. Gwon, H. et al. Flexible energy storage devices based on graphene paper. Energy Environ. Sci. 4, 1277-1283 (2011).

8. Lang, X., Hirata, A., Fujita, T. \& Chen, M. Nanoporous metal/oxide hybrid electrodes for electrochemical supercapacitors. Nat. Nanotech. 6, 232-236 (2011).

9. Bae, J. et al. Fiber Supercapacitors made of nanowire-fiber hybrid structures for wearable/flexible energy Ssorage. Angew. Chem. Int. Ed. 50, 1683-1687 (2011).

10. Chmiola, J., Largeot, C., Taberna, P.-L., Simon, P. \& Gogotsi, Y. Monolithic carbidederived carbon films for micro-supercapacitors. Science 328, 480-483 (2010).

11. Futaba, D. N. et al. Shape-engineerable and highly densely packed single-walled carbon nanotubes and their application as super-capacitor electrodes. Nat. Mater. 5, 987-994 (2006).

12. Eda, G., Fanchini, G. \& Chhowalla, M. Large-area ultrathin films of reduced graphene oxide as a transparent and flexible electronic material. Nat. Nanotech. 3, 270-274 (2008).

13. Liu, J. et al. $\mathrm{Co}_{3} \mathrm{O}_{4}$ nanowire@ $\mathrm{MnO}_{2}$ ultrathin nanosheet core/shell arrays: a new class of high-performance pseudocapacitive materials. Adv. Mater. 23, 2076-2081 (2011)

14. Wang, X. et al. Three-dimensional hierarchical $\mathrm{GeSe}_{2}$ nanostructures for high performance flexible all-solid-state supercapacitors. Adv. Mater. 25, 1479-1486 (2013).

15. $\mathrm{Yu}, \mathrm{G}$. et al. Solution-processed graphene $/ \mathrm{MnO}_{2}$ nanostructured textiles for high-performance electrochemical capacitors. Nano Lett. 11, 2905-2911 (2011).

16. Brezesinski, T., Wang, J., Tolbert, S. H. \& Dunn, B. Ordered mesoporous $\alpha$ $\mathrm{MoO}_{3}$ with iso-oriented nanocrystalline walls for thin-film pseudocapacitors. Nat. Mater. 9, 146-151 (2010)

17. Wang, H., Casalongue, H. S., Liang, Y. \& Dai, $\mathrm{H} . \mathrm{Ni}(\mathrm{OH})_{2}$ nanoplates grown on graphene as advanced electrochemical pseudocapacitor materials. J. Am. Chem. Soc. 132, 7472-7477 (2010).

18. Yoo, J. J. et al. Ultrathin planar graphene supercapacitors. Nano Lett. 11, 1423-1427 (2011)

19. Feng, J. et al. Metallic few-layered $\mathrm{VS}_{2}$ ultrathin nanosheets: high twodimensional conductivity for in-plane supercapacitors. J. Am. Chem. Soc. 133, 17832-17838 (2011)

20. Xie, J. et al. Layer-by-layer $\beta-\mathrm{Ni}(\mathrm{OH})_{2} /$ graphene nanohybrids for ultraflexible all-solid-state thin-film supercapacitors with high electrochemical performance. Nano Energy 2, 65-74 (2013).

21. Wu, C. \& Xie, Y. Promising vanadium oxide and hydroxide nanostructures: from energy storage to energy saving. Energy Environ. Sci. 3, 1191-1206 (2010).
22. Dupré, N. et al. Positive electrode materials for lithium batteries based on $\mathrm{VOPO}_{4}$. Solid State Ionics 140, 209-221 (2001).

23. Azmi, B. M., Ishihara, T., Nishiguchi, H. \& Takita, Y. Vanadyl phosphates of $\mathrm{VOPO}_{4}$ as a cathode of Li-ion rechargeable batteries. J. Power Sources 119-121, 273-277 (2003)

24. Azmi, B. M., Ishihara, T., Nishiguchi, H. \& Takita, Y. Cathodic performance of $\mathrm{VOPO}_{4}$ with various crystal phases for Li ion rechargeable battery. Electrochim. Acta 48, 165-170 (2002).

25. Park, N.-G., Kim, K. M. \& Chang, S. H. Sonochemical synthesis of the high energy density cathode material $\mathrm{VOPO}_{4} \cdot 2 \mathrm{H}_{2} \mathrm{O}$. Electrochem. Commun. 3, 553-556 (2001).

26. Luo, J.-Y., Cui, W.-J., He, P. \& Xia, Y.-Y. Raising the cycling stability of aqueous lithium-ion batteries by eliminating oxygen in the electrolyte. Nat. Chem. 2 760-765 (2010).

27. Fan, Z. et al. Asymmetric supercapacitors based on graphene $/ \mathrm{MnO}_{2}$ and activated carbon nanofiber electrodes with high power and energy density. Adv. Funct. Mater. 21, 2366-2375 (2011)

28. Beneš, L., Melánová, K., Svoboda, J. \& Zima, V. Intercalation chemistry of layered vanadyl phosphate: a review. J. Incl. Phenom. Macro. 73, 33-53 (2012).

29. Markovitch, O. \& Agmon, N. Structure and energetics of the hydronium hydration shells. J. Phys. Chem. A 111, 2253-2256 (2007).

30. Yan, B. et al. Single-crystalline $\mathrm{V}_{2} \mathrm{O}_{5}$ ultralong nanoribbon waveguides. $A d v$. Mater. 21, 2436-2440 (2009).

31. Beneš, L., Melánová, K., Trchová, M., Čapková, P. \& Matějka, P. Water/Ethanol displacement reactions in vanadyl phosphate. Eur. J. Inorg. Chem. 12, 2289-2294 (1999).

32. Yan, Z. et al. Growth of bilayer graphene on insulating substrates. ACS Nano $\mathbf{5}$, 8187-8192 (2011).

33. Zhu, Y. et al. Graphene and graphene oxide: synthesis, properties, and applications. Adv. Mater. 22, 3906-3924 (2010).

34. Malard, L. M., Pimenta, M. A., Dresselhaus, G. \& Dresselhaus, M. S. Raman spectroscopy in graphene. Phys. Rep. 473, 51-87 (2009).

35. Wang, G. et al. LiCl/PVA gel electrolyte stabilizes vanadium oxide nanowire electrodes for pseudocapacitors. ACS Nano 6, 10296-10302 (2012).

36. Jung, H. Y., Karimi, M. B., Hahm, M. G., Ajayan, P. M. \& Jung, Y. J. Transparent, flexible supercapacitors from nano-engineered carbon films. Sci. Rep. 2, 773-777 (2012).

37. Peng, L. et al. Ultrathin Two-Dimensional $\mathrm{MnO}_{2} /$ Graphene hybrid nanostructures for high-performance, flexible planar supercapacitors. Nano Lett. 13, 2151-2157 (2013).

38. Lu, Q. et al. Supercapacitor electrodes with high-energy and power densities prepared from nonolithic $\mathrm{NiO} / \mathrm{Ni}$ nanocomposites. Angew. Chem. Int. Ed. 123 6979-6982 (2011).

39. Yamamoto, N., Hiyoshi, N. \& Okuhara, T. Thin-layered sheets of $\mathrm{VOHPO}_{4} \cdot 0.5 \mathrm{H}_{2} \mathrm{O}$ prepared from $\mathrm{VOPO}_{4} \cdot 2 \mathrm{H}_{2} \mathrm{O}$ by intercalation exfoliation - reduction in alcohol. Chem. Mater. 14, 3882-3888 (2002).

40. Marcano, D. C. et al. Improved synthesis of graphene oxide. ACS Nano 4, 4806-4814 (2010).

41. Nkovich, S. et al. Synthesis of graphene-based nanosheets via chemical reduction of exfoliated graphite oxide. Carbon 45, 1558-1565 (2007).

\section{Acknowledgements}

C.W. and Y.X. acknowledge financial support from the National Basic Research Program of China (No. 2009CB939901), National Natural Science Foundation of China (No. 21222101, 11074229, 11132009, 11079004, J1030412), Chinese Academy of Science (XDB01020300), Program for New Century Excellent Talents in University. G.Y. acknowledges the start-up funding support from Cockrell School of Engineering at The University of Texas at Austin.

\section{Author contributions}

C.W., Y.X. and G.Y. conceived the idea, co-wrote the paper and supervised the whole project. C.W., X.L. and L.P., experimentally realized the idea and wrote the paper X.L., L.P., X.K. and X.P. carried out the examples synthesis, characterization and data analysis; J.H. performed the atomic force microscopy investigation. All the authors discussed the results, commented on and revised the manuscript.

\section{Additional information}

Supplementary Information accompanies this paper at http://www.nature.com/ naturecommunications

Competing financial interests: The authors declare no competing financial interests.

Reprints and permission information is available online at http://npg.nature.com/ reprintsandpermissions/

How to cite this article: $\mathrm{Wu}, \mathrm{C}$. et al. Two-dimensional vanadyl phosphate ultrathin nanosheets for high energy density and flexible pseudocapacitors. Nat. Commun. 4:2431 doi: $10.1038 /$ ncomms3431 (2013). 